\title{
Somatotopic organization of the primate basal ganglia
}

\author{
Atsushi Nambu* \\ Division of System Neurophysiology, National Institute for Physiological Sciences, Okazaki, Japan
}

Edited by:

Jose L. Lanciego, University of

Navarra, Spain

Reviewed by:

Lydia Kerkerian-Le Goff, CNRS-

Mediterranean University, France

Robert S. Turner, University of

Pittsburgh, USA

\section{*Correspondence:}

Atsushi Nambu, Division of System

Neurophysiology, National Institute for

Physiological Sciences, 38

Nishigonaka, Myodaiji, Okazaki,

444-8585, Japan.

e-mail: nambu@nips.ac.jp
Somatotopic organization is a fundamental and key concept to understand how the corticobasal ganglia loop works. It is also indispensable knowledge to perform stereotaxic surgery for movement disorders. Here I would like to describe the somatotopic organization of the basal ganglia, which consist of the striatum, subthalamic nucleus, globus pallidus, and substantia nigra. Projections from motor cortical regions representing different body parts terminate in different regions of these nuclei. Basal ganglia neurons respond not only to the stimulation of the corresponding regions of the motor cortices, but also to active and passive movements of the corresponding body parts. On the basis of these anatomical and physiological findings, somatotopic organization can be identified in the motor territories of these nuclei in the basal ganglia. In addition, projections from functionally interrelated cortical areas partially converge through the cortico-basal ganglia loop, but nevertheless the somatotopy is still preserved. Disorganized somatotopy may explain, at least in part, the pathophysiology of movement disorders, such as Parkinson's disease and dystonia.

Keywords: striatum, subthalamic nucleus, globus pallidus, substantia nigra, somatotopy, movement disorders

\section{INTRODUCTION}

Somatotopic organization in the cerebral cortex, especially in the primary motor and primary somatosensory cortices, is a well-known and fundamental concept to understand the functions of these areas. Each nucleus of the basal ganglia also shows somatotopy, but it has received little attention. Somatotopy of the basal ganglia is disorganized in movement disorders, suggesting its pathophysiological significance. Knowledge on somatotopy of the human basal ganglia is also indispensable to identify the location of the tip of electrodes during stereotaxic surgery for movement disorders. In this article, I would like to describe the somatotopic organization of the basal ganglia comprehensively and in detail. Although the description is mainly based on monkey studies, it should be applicable to the human basal ganglia because the basal ganglia of non-human primates and humans share a number of common properties, despite their size difference.

\section{BASIC CIRCUITRY OF THE BASAL GANGLIA}

The basal ganglia are a group of sub-cortical nuclei, and are composed of the striatum, pallidum, subthalamic nucleus (STN), and substantia nigra ( $\mathrm{SN}$ ). The striatum can be classified into the caudate nucleus, putamen, and ventral striatum. The pallidum can be divided into the external (GPe) and internal (GPi) segments of the globus pallidus and ventral pallidum (VP). The $\mathrm{SN}$ is composed of pars reticulata $(\mathrm{SNr})$ and pars compacta $(\mathrm{SNc})$. Among these nuclei, the striatum and STN are input stations of the basal ganglia. The striatum receives inputs from the entire cerebral cortex except the primary visual cortex, and the STN receives inputs mainly from the frontal cortex. On the other hand, the GPi and SNr serve as the output nuclei of the basal ganglia, and project outside the basal ganglia. The GPe connects input stations to the output nuclei. The SNc is composed of dopaminergic neurons, which project widely to the whole basal ganglia, especially to the striatum, and modulate their activity.
Cortical information received in the input stations is transferred to the output nuclei through the following three pathways (Figure 1; Alexander and Crutcher, 1990a; Nambu et al., 2002b).

Direct pathway: Striatal neurons expressing substance $\mathrm{P}$ receive cortical inputs and project directly to the GPi/SNr.

Indirect pathway: Striatal neurons expressing enkephalin receive cortical inputs and project polysynaptically to the GPi/SNr by way of the GPe and STN.

Hyperdirect pathway: STN neurons receive direct cortical inputs and project to the GPi/SNr. This pathway transfers cortical excitation faster to the GPi/SNr than the direct and indirect pathways.

Information originating from the frontal cortex is processed through these three pathways, and mainly returns to the frontal cortex through the thalamus, thus forming the cortico-basal ganglia loop. Some information is transferred to the brainstem from the output nuclei (Alexander and Crutcher, 1990a).

The primary motor cortex (MI), supplementary motor area (SMA) and premotor cortex (PM) are classically defined motor cortices. In addition, the pre-SMA in the mesial side of the hemisphere anterior to the SMA and the cingulate motor areas (CMA) in the cingulate sulcus have been identified (Picard and Strick, 2001). The PM is not homogeneous and was originally divided into dorsal and ventral parts (PMd and PMv), and is now further subdivided into rostral and caudal parts (PMdr, PMdc, PMvr, and PMvc). The CMA is divided into rostral and caudal parts (CMAr and CMAc). Among them, the most rostral motor cortices, such as pre-SMA, PMvr, PMdr, and CMAr, receive inputs from the frontal association cortex and send outputs to the more caudal motor cortices, such as SMA, PMvc, PMdc, and CMAc (Takada et al., 2004). Most of these motor cortices, especially SMA, PMvc, PMdc, CMAc, and MI have their own somatotopy. 
There have been two opposing views concerning how information originating from different cortical areas or different somatotopic regions is processed through the basal ganglia (Figure 2; Parent and Hazrati, 1995). One is the parallel processing hypothesis (Alexander et al., 1986; Hoover and Strick, 1993; Strick et al., 1995) proposing that information from different cortical areas is processed independently in the different parts of the basal ganglia (Figure 2A). The other is the information convergence hypothesis (Percheron and Filion, 1991; Percheron et al., 1994) proposing that information from different cortical areas converges and is integrated in the basal ganglia (Figure 2B). Recent studies suggest that both parallel processing and information convergence occur (Figure 2C). Information from cortical areas whose functions are distinct from each other terminates in the different regions in the basal ganglia. On the other hand, information from cortical areas whose functions are close to each other tends to converge in the basal ganglia. For example, projections from the motor, oculomotor, prefrontal, and limbic cortices terminate in different regions in the striatum. These striatal regions project to different regions of other basal ganglia nuclei. Thus, each nucleus of the

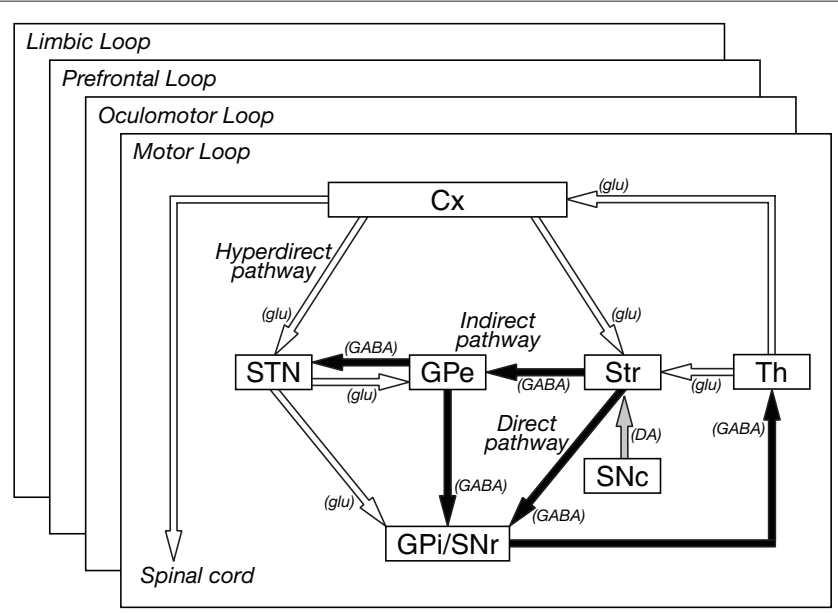

FIGURE 1 | Basic circuitry of the basal ganglia. Open and filled arrows indicate excitatory and inhibitory projections, respectively. $\mathrm{Cx}$, cerebral cortex: DA, dopamine, GABA, gamma-aminobutyric acid; glu, glutamate; GPe and $\mathrm{GPi}$, external and internal segments of the globus pallidus; $\mathrm{SNc}$, substantia nigra pars compacta; SNr substantia nigra pars reticulata; STN, subthalamic nucleus; Str, striatum; Th, thalamus. Modified from Nambu et al. (2002b). basal ganglia can be segregated into motor, oculomotor, prefrontal, and limbic territories, and cortico-basal ganglia loops are composed of several independent and functionally distinct, but homological loops: motor, oculomotor, prefrontal, and limbic loops (Figure 1). Each loop controls brain functions independently (Alexander et al., 1986; Parent, 1990). Inside the motor loop, projections from the MI, SMA, and PM partly converge in the striatum, while projections from the MI and pre-SMA project to distinct regions of the striatum. Somatotopy is also well defined in each nucleus of the basal ganglia, and information from different body parts of the somatotopy is well preserved through cortico-basal ganglia loops.

\section{METHODS TO IDENTIFY SOMATOTOPY}

Somatotopy of the basal ganglia reflects input and output connections of each nucleus, and can be investigated in several ways. The most basic method is an anatomical method examining fiber connections with other brain areas whose somatotopy is clearly identified. For example, anterograde tracers are injected into the orofacial, forelimb, and hindlimb regions of the MI, and then terminals in the striatum and STN are observed. Transsynaptic anterograde and retrograde tracing can be performed using herpes simplex virus (anterograde or retrograde) and rabies virus (retrograde) as tracers. Fiber connections can also be investigated by electrophysiological methods. Stimulation of the MI induces responses in the corresponding regions in the striatum, STN, GPe, and GPi.

Another useful electrophysiological method is recording neuronal activity in behaving animals. Neurons in the basal ganglia change activity during active movements of the corresponding body parts. These neurons usually respond to passive movements of the corresponding body parts as well, such as manipulations of joints and muscle palpations. Applying microstimulation through recording electrodes in some nuclei of the basal ganglia can induce movements of the corresponding body parts, although more pulses are necessary compared with that for intracortical microstimulation.

In the following sections, somatotopy in each nucleus of the basal ganglia will be discussed. "Cartoons" representing somatotopy will be drawn for each nucleus. However, they are metaphors, and readers should not take them too literally. For example, in Figure 5, the orofacial, forelimb, and hindlimb regions are represented in this order along the ventral-to-dorsal axis of the globus pallidus, but it is not known whether each finger is distinctly and orderly represented (Hamada et al., 1990).
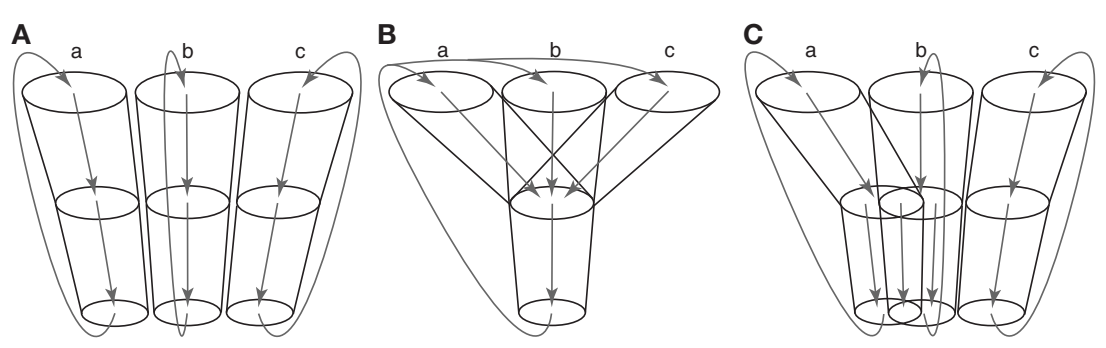

FIGURE 2 | Information processing in the basal ganglia. (A) Paralle/ processing hypothesis. Information originating from different areas $(a, b, c)$ of the cerebral cortex is processed independently in the different parts of the basal ganglia, and returns to the original cortical areas. (B) Information convergence hypothesis. Information originating from different cortical areas converges and is integrated in the basal ganglia, and integrated information returns to all the cortical areas. (C) Intermediate hypothesis between parallel processing and information convergence hypotheses, which is supported by recent studies. 


\section{STRIATUM}

The striatum, as an input station of the basal ganglia, receives excitatory inputs from all areas of the cerebral cortex except the primary visual cortex. The caudal aspect of the putamen, which is posterior to the anterior commissure, is considered to be the motor territory and shows clear somatotopy (Figure 3A). Distribution patterns of labeling in the striatum were observed after injection of anterograde tracers into the orofacial, forelimb, and hindlimb regions of the MI and SMA (Künzle, 1975; Flaherty and Graybiel, 1993; Takada et al., 1998b). Labeling consisted of dense and diffuse projection regions as recently proposed (Haber et al., 2006). The dense terminals were found in the lateral part (MI territory) after injection into the MI, and in the medial part (SMA territory) after injection into the SMA. The orofacial, forelimb, and hindlimb regions of the MI project to the ventral to dorsal parts of the lateral putamen. The corresponding regions of the SMA project to the ventral to dorsal parts of the medial putamen, which are mediodorsal to the MI territory. Therefore, the putamen has two sets of somatotopic representations in the medial and lateral parts. The diffuse terminals from the MI extend to the dorsomedial portion, and those from the SMA extend to the ventrolateral portion. Thus, the projections from the orofacial, forelimb, and hindlimb regions of the MI and those from the corresponding regions of the SMA converge in the medio-lateral central zone that occupies onequarter of each territory. The forelimb region is widely represented in the MI territory. The proximal regions (elbow and shoulder) are located in the mediodorsal part, and the distal regions (wrist and digits) are located in the ventrolateral part (Tokuno et al., 1999).

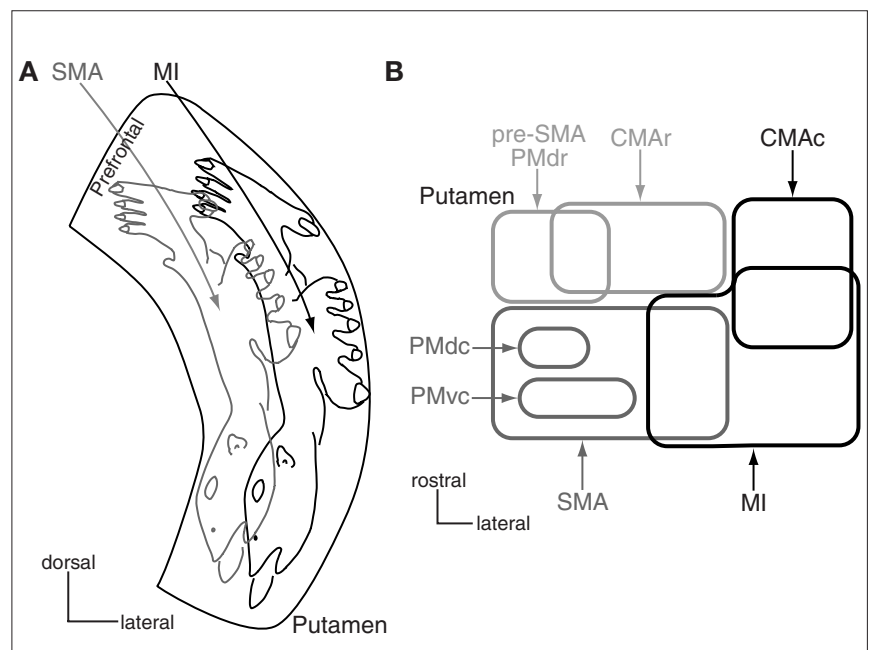

FIGURE 3 | Somatotopy of the putamen. (A) Somatotopy of the putamen is schematically shown in a frontal section. In the caudal aspect of the putamen, the lateral part receives somatotopic inputs from the primary motor cortex (MI), and the medial part from the supplementary motor area (SMA). The somatotopy in the SMA territory is located dorsomedially to that in the MI territory. Projections from the orofacial, forelimb and hindlimb regions of the $\mathrm{MI}$ and SMA converge in the medio-lateral central zone in the putamen. The most dorsomedial part receives inputs from the prefrontal cortex. Modified from Nambu et al. (2002a) (B) Input from motor cortices to the putamen is schematically shown in a horizontal section. CMAc and CMAr, caudal and rostral parts of the cingulate motor area; PMdc, PMdr, and PMvc, caudal part of dorsal premotor cortex, rostral part of dorsal premotor cortex, and caudal part of ventral premotor cortex. Modified from Takada et al. (2001).
This somatotopy reflects not only cortical inputs revealed by conventional tracers, but also putaminal outputs to the cortex through the direct and indirect pathways. Injection of rabies virus into the MI resulted in retrograde transsynaptic labeling of neurons in the putamen, which showed similar somatotopic organization, especially in its lateral side (Miyachi et al., 2006). Moreover, this study also showed no labeling of neurons in the SMA territory of the putamen, suggesting that the pathways originating from the MI territory of the putamen and from the SMA territory are independent of each other in the basal ganglia. The motor territory of the putamen also receives topographic inputs from the motor thalamus and centromedian and parafascicular nuclei, which are reciprocally connected with motor cortices (Nakano et al., 1990; Sadikot et al., 1992; McFarland and Haber, 2000; Jones, 2007). These projections are also considered to be somatotopically organized.

The somatotopy in the putamen is also confirmed by electrophysiological methods (Nambu et al., 2002a). Cortical stimulation of the forelimb regions of the MI and SMA orthodromically activates projection neurons in the corresponding MI (lateral) and SMA (medial) territories of the putamen, respectively, at a latency of 10-15 ms. Putaminal neurons in the central zones are activated by the stimulation of both the MI and SMA, and thus, convergence from the MI and SMA occurs at a single neuronal level. Putaminal neurons in the MI and SMA territories are activated by passive and/or active movements of the corresponding body parts on the contralateral side (Alexander and DeLong, 1985; Alexander and Crutcher, 1990b; Nambu et al., 2002a). However, putaminal neurons in the MI territory and those in the SMA territory show different activity patterns during task performance. Putaminal neurons in the MI territory are closely related to movements themselves, while neurons in the SMA territory are activated not only by movements themselves, but also during delay periods. Such activity differences of putaminal neurons seem to reflect the activity patterns of MI and SMA neurons that give rise to cortico-striatal projections. Microstimulation in the MI territory of the putamen produces movements of the corresponding body parts, while that in the SMA territory does not (Alexander and DeLong, 1985; Nambu et al., 2002a). The probable pathway for inducing movements by microstimulation is the direct pathway. Stimulation of the striatum may excite direct pathway neurons, inhibit GPi and finally disinhibit thalamic and cortical activity. The microstimulation studies suggest that putaminal neurons in the MI and SMA territories project independently to different territories in the nucleus of the basal ganglia, and that somatotopy is preserved through the basal ganglia circuitry.

Striatal projection neurons are classified into direct and indirect pathway neurons on the basis of the difference in receptors, peptides, and targets. The two groups of neurons may represent similar somatotopy and show similar activity patterns during task performance. The striatum also contains interneurons. Although cholinergic interneurons receive common cortical inputs with neighboring projection neurons, they show reward-related activity (Aosaki et al., 1995), which is different from that of neighboring projection neurons. Parvalbumin (PV)-positive GABAergic interneurons also receive cortical inputs and are thought to regulate the activity of projection neurons through feed-forward inhibition (Tepper et al., 2008). PV-positive interneurons showed task-related activity (Gage et al., 2010), suggesting that they share similar cortical inputs with neighboring 
projection neurons. Activity patterns of other interneurons during task performance remain to be studied. The striatum is classified into $\mu$-opiate receptor-rich patch compartment (or striosome) and matrix compartment (Graybiel, 1990), but the relationship between somatotopy and patch-matrix organization is unclear.

Other motor cortices also project to the striatum (Figure 3B; Takada et al., 1998a,b, 2001; Inase et al., 1999; Tachibana et al., 2004). The highest motor cortices, such as pre-SMA, PMdr, and CMAr, project to the anterior part of the striatum, especially to the bridge region connecting the caudate nucleus and putamen. The forelimb regions of the PMdc and PMvc project to two independent regions in the SMA territory of the putamen. On the other hand, the CMAc, which shows activity similar to that of the MI, projects to the MI territory. Projections from the primary somatosensory cortex also project to the MI territory (Flaherty and Graybiel, 1993). The projection patterns seem to obey the following rules: The motor cortices whose functions are distinct project to the different regions of the striatum, whereas the motor cortices whose functions are similar project to the common striatal regions in a convergent manner. The prefrontal cortex projects to the rostral part of the putamen anterior to the anterior commissure and the head of the caudate nucleus (prefrontal territory of the striatum), and the limbic cortex projects to the ventral striatum (limbic territory; Selemon and Goldman-Rakic, 1985; Haber et al., 1990; Parent, 1990). Eye movement-related neurons are located in the central part of the caudate nucleus (oculomotor territory; Hikosaka et al., 1989).

\section{SUBTHALAMIC NUCLEUS (STN)}

The STN, another input station of the basal ganglia, receives cortical inputs from the frontal lobe. The dorsal part of the STN is the motor territory and shows somatotopic organization (Figure 4A; Monakow et al., 1978; Nambu et al., 1996). The MI projects to the lateral part (MI territory), and the SMA projects to the medial part (SMA territory). The orofacial, forelimb, and hindlimb regions of the MI project to the lateral to medial parts of the lateral STN, while those of the SMA project to the medial to lateral parts of the medial STN. Therefore, two sets of somatotopic representations, which are mirror images of each other, are represented in the lateral and medial parts of the STN. The MI also partly projects to the somatotopically corresponding body parts in the SMA territory, and the SMA partly projects to the MI territory, vice versa. Thus, inputs from the MI and SMA partly converge in the STN. The forelimb regions of the PMdc and PMvc also project to the forelimb region of the SMA territory (Figure 4B; Nambu et al., 1997). The somatotopy of the STN reflects not only input organization, but also output organization, because similar somatotopy is observed after transneuronal retrograde labeling of rabies virus by its injection into the MI (Miyachi et al., 2006).

The somatotopy of the STN has also been confirmed by electrophysiological methods. Cortical stimulation of the MI and SMA induces a short latency excitation and a subsequent long latency excitation (Nambu et al., 2000), which are mediated by the corticoSTN (hyperdirect) and cortico-striato-GPe-STN (indirect) pathways, respectively. By observing cortically evoked responses, similar somatotopy can be drawn, with some neurons receiving convergent inputs from the MI and SMA. STN neurons in the MI territory change their activity (mostly excitation) in relation to active or passive movements of the corresponding body parts on the contralateral side (DeLong et al., 1985; Wichmann et al., 1994). STN neurons in the SMA territory may also show task-related activity. Microstimulation in the MI and SMA territories does not evoke movements, while that in the most lateral part of the STN often evokes movements probably because of the current spread to the internal capsule (Wichmann et al., 1994).

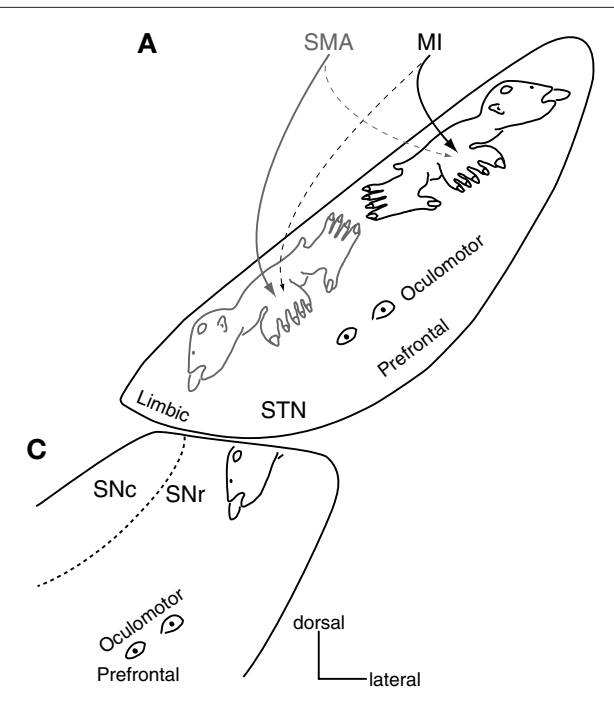

B

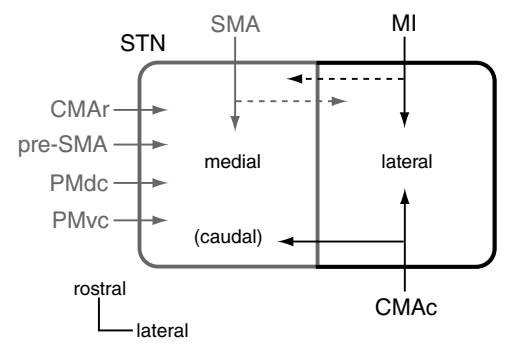

FIGURE 4 | Somatotopy of the subthalamic nucleus (STN) and substantia nigra (SN). (A) Somatotopy of the STN is shown in a frontal section. In the dorsal part of the STN, the lateral part receives somatotopic inputs from the MI, and the medial part from the SMA. The MI also projects partly to the medial part, and the SMA to the lateral part. Ventral to the motor territory, there exist the oculomotor and prefrontal territories. The most medial part is occupied by the limbic territory. Modified from Nambu et al. (2002b). (B) Input from motor cortices to the STN is schematically shown in a horizontal section. Modified from Takada et al. (2001). (C) Somatotopy of the SNr is shown in a frontal section. The orofacial region of the SNr is a continuation of that of the GPi (see Figure 5). Ventral to the motor territory, there exist the oculomotor and prefrontal territories. 
Concerning other motor cortical inputs, the CMAc projects to the MI territory of the STN, and the pre-SMA and CMAr project to the SMA territory (Figure 4B; Inase et al., 1999; Takada et al., 2001). Thus, more convergence may occur in the cortico-STN projections than in the cortico-striatal projections (compare Figure 4B with Figure 3B), suggesting that the hyperdirect pathway assembles information from more wide areas of the motor cortices than the direct and indirect pathways do. Ventral to the motor territory in the STN, there exist the oculomotor territory (Matsumura et al., 1992) and the prefrontal territory (Monakow et al., 1978; Parent, 1990; Figure 4A). The most ventromedial part of the STN is occupied by the limbic territory (Parent, 1990).

\section{EXTERNAL AND INTERNAL SEGMENTS OF THE GLOBUS PALLIDUS (GPe AND GPi)}

The motor territory of the striatum (i.e., the caudal aspect of the putamen) projects to the ventral two-thirds of the caudal GPe and $\mathrm{GPi}$, and thus, these areas are the motor territories of the globus pallidus (Smith and Parent, 1986; Parent, 1990) that show somatotopic organization (Figure 5). In GPe/GPi neurons, cortical stimulation evokes a triphasic response composed of early excitation, inhibition, and late excitation, which are mediated by the cortico-STNGPe/GPi (cortico-STN-GPi: hyperdirect), cortico-striato-GPe/GPi (cortico-striato-GPi: direct), and cortico-striato-GPe-STN-GPe/ GPi (cortico-striato-GPe-STN-GPi: indirect) pathways, respectively (Nambu et al., 2000; Kita et al., 2004; Tachibana et al., 2008). The somatotopy in the GPe/GPi can be drawn by observing responses evoked by the stimulation of the MI and SMA. Neurons responding to the orofacial, forelimb, and hindlimb regions of the MI are located along the ventral-to-dorsal axis in the GPe and GPi (MI territory, Figure 5; Yoshida et al., 1993). Neurons responding to the corresponding regions of the SMA are also located along the ventral-to-dorsal axis, but in more rostral and dorsal parts of the GPe/GPi (SMA territory). Stimulation of the PM also evokes responses in the SMA territory. GPe/GPi neurons rarely respond to cortical stimulation of multiple body parts, and thus, the orofacial, forelimb, and hindlimb regions of GPe/GPi are clearly and distinctly identified. On the other hand, many neurons respond to the stimulation of both the MI and SMA, and the somatotopic representation in the MI territory and that in the SMA territory are partly fused in the rostro-caudal central zone. Most GPe/GPi neurons show triphasic responses evoked by cortical stimulation, suggesting that the hyperdirect, direct, and indirect pathways originating from a certain body region in the cortex converge at a single GPe/GPi neuronal level.

The above-mentioned somatotopy is also supported by anatomical studies. The injection of anterograde tracers into the MI, SMA, and convergent territories in the putamen revealed the terminals in the GPe/GPi (Kaneda et al., 2002). Terminals from the SMA territory of the putamen are located more anterior and dorsal to those from the MI territory. The convergent territory of the putamen projects to the area in-between, and these three projection territories do not overlap. Transsynaptic anterograde and retrograde labeling studies by injecting herpes simplex virus into the MI reported similar results (Hoover and Strick, 1993, 1999; Strick et al., 1995; Akkal et al., 2007), although there is some discrepancy, such as that the PMv territory of the GPe/GPi is located ventrally to the MI territory. Dendritic fields of GPe/GPi neurons extend widely in the direction perpendicular to the striato-pallidal fibers, and this is considered to be the basis for information convergence (Percheron et al., 1984; Yelnik et al., 1984). However, somatotopic organization through the striato-pallidal projections is well preserved as described above.

Neurons in the MI and SMA territories of the GPe/GPi change their activity in relation to active and passive movements of the corresponding body parts on the contralateral side (DeLong, 1971; Georgopoulos et al., 1983; DeLong et al., 1985; Hamada et al., 1990). However, response patterns are different between these territories. Neurons in the MI territory show movement-related activity, while those in the SMA territory show delay-related activity (Nambu et al., 1990). On the other hand, response patterns in the GPe and GPi neurons during task performance are very similar. Microstimulation in the GPe/GPi does not induce any movements.

The prefrontal territory of the striatum projects to the rostral GPe and dorsal one-third of the caudal GPe/GPi, and thus, these areas are the prefrontal territory (Smith and Parent, 1986; Parent, 1990). Ventral striatum projects to the VP, the most rostral part of the GPe and the most medial part of the GPi, and thus, these areas correspond to the limbic territory (Haber et al., 1990; Parent, 1990).

\section{SUBSTANTIA NIGRA (SN)}

The SNr and GPi are the output nuclei of the basal ganglia and considered to be a continuum, which is divided into the $\mathrm{SNr}$ and GPi by the internal capsule. The motor territory of the striatum projects to the dorsal one-third of the $\mathrm{SNr}$, and thus, this area is considered to be the motor territory of the SNr (Figure 4C; Smith and Parent, 1986; Parent, 1990). Neurons in the dorsolateral part

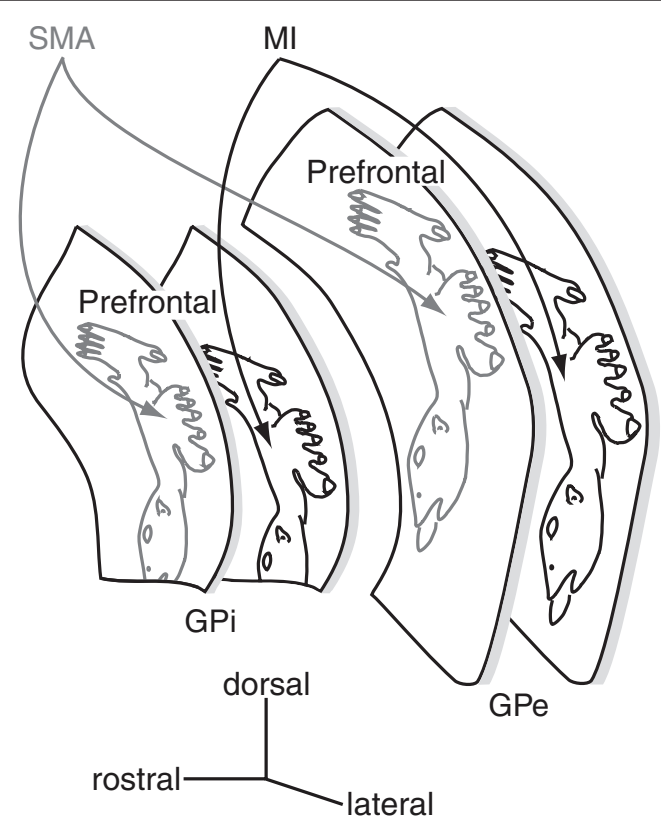

FIGURE 5 | Somatotopy of the external and internal segments of the globus pallidus (GPe and GPi). The GPe and GPi have their own somatotopic representations. The somatotopy in the SMA territory is located rostrodorsally to that in the MI territory. Two territories overlap partly in the rostro-caudal zone. Dorsal one-third of the GPe/GPi is occupied by the prefrontal territory. 
of this area respond to the stimulation of the MI, especially to that of the orofacial region, and change their activity in relation to active or passive movements of the orofacial region (DeLong et al., 1983; Kitano et al., 1998). The orofacial region of the $\mathrm{SNr}$ is considered to be a continuation of the orofacial region of the GPi (see Figures 4C and 5). SNr neurons in the part ventral to the orofacial region receive inputs from the SMA territories of the putamen, and change their activity during task performance. However, the somatotopy is not clearly organized, and their activity is not so distinct compared to that of GPi neurons (Wichmann and Kliem, 2004). The prefrontal territory of the striatum projects to the rostromedial two-thirds of the SNr (Smith and Parent, 1986) that also include the oculomotor territory (Hikosaka and Wurtz, 1983; Figure 4C). The limbic territory of the striatum projects to the most medial part of the $\mathrm{SNr}$ (Haber et al., 1990).

The SNc is composed of dopaminergic neurons, and projects to the striatum and other basal ganglia nuclei. Dopaminergic projections from the SNc to the striatum display weak topography, and the terminal fields of a single dopaminergic neuron are large (Parent et al., 1983; Parent, 1990; Matsuda et al., 2009). SNc neurons do not respond to active or passive body part movements, but respond to novel sensory stimuli and/or rewards (DeLong et al., 1983; Schultz and Romo, 1990). Recent studies suggest that SNc neurons code the difference between the expected reward and the real reward (a temporal difference error in reinforcement learning). These observations suggest that the SNc has no clear somatotopy.

\section{THALAMUS}

The motor thalamus is a target structure of the basal ganglia, and also shows somatotopy (Figure 6). Subnuclei located in the rostral part of the motor thalamus receive inputs from the basal ganglia. The oral part of the ventrolateral nucleus (VLo) and the principal part of the ventroanterior nucleus (VApc) receive inputs from the GPi. The medial part of the ventrolateral nucleus (VLm) and the magnocellular part of the ventroanterior nucleus (VAmc) receive inputs from the SNr. On the other hand, subnuclei located in the caudal part, such as the oral part of the ventroposterolateral nucleus (VPLo), the caudal part of the ventrolateral nucleus (VLc) and area X, receive cerebellar inputs (Jones, 2007). Thus, projections from the SNr, GPi and cerebellar nuclei terminate in the rostral to caudal parts of the motor thalamus, and the overlap of their terminals is minimal. The VApc, VLo, VPLo, and VLc project to the motor cortices, and thus, most of the motor cortices receive inputs from both the basal ganglia and the cerebellum through the motor thalamus (Jones, 2007). The MI receives basal ganglia inputs through the VLo, and cerebellar inputs through the VPLo (Holsapple et al., 1991).

The VLo and VPLo display clear somatotopic organization (Figure 6). The orofacial, forelimb, and hindlimb regions are represented in the medial to lateral parts (Asanuma et al., 1983; Vitek et al., 1994). VLo neurons change their activity in relation to active movements of the corresponding body parts (Anderson and Turner, 1991; Nambu et al., 1991; Vitek et al., 1994). However, sensory inputs are not clearly identified, and the microstimulation in the VLo does not induce any movements (Buford et al., 1996; Vitek et al., 1996). On the other hand, VPLo neurons respond clearly to active and passive movements of discrete body parts (one to several joints) on the contralateral side. Microstimulation in the VPLo induces movements in the corresponding body parts, contralateral to the stimulation side. The somatotopy can also be confirmed by the anatomical study of the thalamo-cortical projections (Asanuma et al., 1983; Holsapple et al., 1991). Therefore, the thalamus has at least two sets of somatotopic representations: one in the GPireceiving region (VLo) and the other in the cerebellar-receiving region (VPLo).

\section{FUNCTIONAL SIGNIFICANCE OF THE SOMATOTOPY}

Each nucleus of the basal ganglia shows clear somatotopic organization, and information originating from cortical regions representing different body parts rarely converges in the cortico-basal ganglia circuitry. These observations suggest that information related to different body parts, such as forelimb and hindlimb, is processed independently through the cortico-basal ganglia loop. On the other hand, information from different but related cortical areas, such as the forelimb regions of the MI and SMA, is processed in both convergent and non-convergent manners. However, the functional roles of such convergence remain to be elucidated.

\section{SOMATOTOPY AND MOVEMENT DISORDERS}

The pathophysiology of movement disorders can be explained by the changes of the firing rates and patterns in the basal ganglia, especially in the GPe, GPi, and STN. In addition, changes in the somatotopy have been reported in movement disorders. In a normal state, GPe and GPi neurons respond specifically to the movement of one direction of a single joint on the contralateral side. On the other hand, GPe/GPi neurons in a Parkinsonian state respond to multiple movements of multiple joints, sometimes of the upper and lower limbs and of both sides (Filion et al., 1988). Loss of functional segregation was also reported in the GPi-receiving thalamus (Pessiglione et al., 2005). Dopamine is considered to contribute to

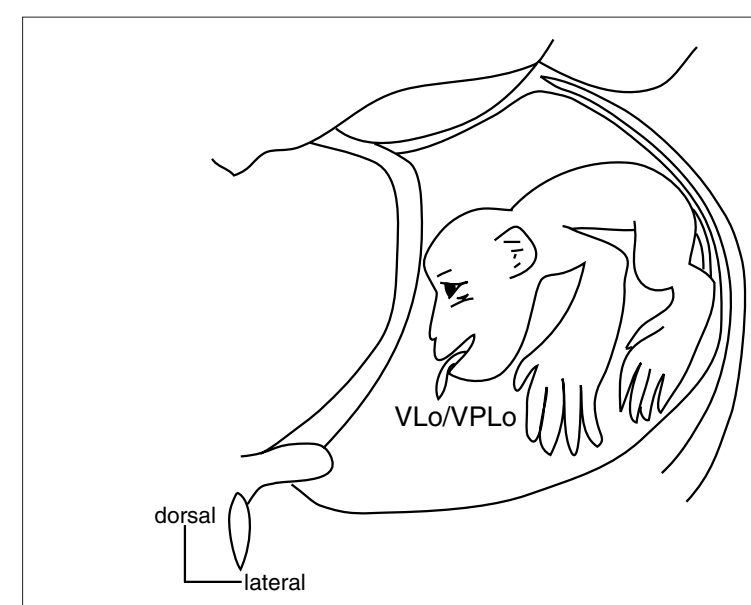

FIGURE 6 | Somatotopy of the thalamus. Somatotopy of the thalamus is shown in a frontal section. Both the oral part of the ventrolateral nucleus of the thalamus (VLO) receiving pallidal projections and the oral part of the ventroposterolateral nucleus of the thalamus (VPLo) receiving cerebellar projections have their own somatotopic representations. Their somatotopic representations are continuous rostrocaudally. Modified from Asanuma et al. (1983). 
isolate information related to movements of specific body parts. Loss of dopamine may induce a crosstalk of information related to different body parts (Bergman et al., 1998).

In dystonia, somatotopy of the basal ganglia is also disorganized (Vitek et al., 1999; Chiken et al., 2008). GPe/GPi neurons respond to stimulation of multiple body parts, and they are intermingled. Dystonic patients show a phenomenon known as "motor overflow." Such a phenomenon may be explained by disorganization of the somatotopy. When patients try to move one body part, for example, a hand, not only the hand region, but also other regions, such as the neck region, of the GPi could be inhibited by somatotopic disorganization. This may lead to unintended movements of other body parts, such as the neck, that accompany intended movements of a hand.

Hemiballism is caused by lesions in the STN, such as a hemorrhage or infarction. Hemiballism in the lower limb is common, while that in the orofacial regions is rare (Carpenter et al., 1950; Hamada and DeLong, 1992). Hemiballism in the upper limb is associated with that in the lower limb. These characteristics can be explained by the mirror image organization of the somatotopy in the STN (Figure 4A; Nambu et al., 1996). It is supposed that the inactivation of both the MI and SMA territories of the corresponding body parts is necessary to produce hemiballism. Small lesions in the central STN affect both lower limb regions of the MI and SMA territories, and thus cause hemiballism in the lower limb. Large lesions affecting both upper limb regions of the MI and SMA territories also affect both lower limb regions, and thus hemiballism in the upper limb is accompanied by that in the lower limb. Lesions affecting both orofacial regions of the MI and SMA territories should be rare because they are remotely located.

Abnormal firing rates and patterns in the motor territory of the basal ganglia cause motor symptoms of the movement disorders. The target of stereotaxic surgery, including deep brain

\section{REFERENCES}

Akkal, D., Dum, R. P., and Strick, P. L. (2007). Supplementary motor area and presupplementary motor area: targets of basal ganglia and cerebellar output. J. Neurosci. 27, 10659-10673.

Alexander, G. E., and Crutcher, M. D. (1990a). Functional architecture of basal ganglia circuits: neural substrates of parallel processing. Trends Neurosci. 13, 266-271.

Alexander, G. E., and Crutcher, M. D. (1990b). Preparation for movement: neural representations of intended direction in three motor areas of the monkey. J. Neurophysiol. 64, 133-150.

Alexander, G. E., and DeLong, M. R. (1985). Microstimulation of the primate neostriatum. II. Somatotopic organization of striatal microexcitable zones and their relation to neuronal response properties. J. Neurophysiol. 53, 1417-1430.

Alexander, G. E., DeLong, M. R., and Strick, P. L. (1986). Parallel organization of functionally segregated circuits linking basal ganglia and cortex. Annu. Rev. Neurosci. 9, 357-381.
Anderson, M. E., and Turner, R. S. (1991). Activity of neurons in cerebellarreceiving and pallidal-receiving areas of the thalamus of the behaving monkey. J. Neurophysiol. 66, 879-893.

Aosaki, T., Kimura, M., and Graybiel, A. M. (1995). Temporal and spatial characteristics of tonically active neurons of the primate's striatum. J. Neurophysiol. 73, 1234-1252.

Asanuma, C., Thach, W. R., and Jones, E. G. (1983). Anatomical evidence for segregated focal groupings of efferent cells and their terminal ramifications in the cerebellothalamic pathway of the monkey. Brain Res. 286, 267-297.

Bergman, H., Feingold, A., Nini, A., Raz, A., Slovin, H., Abeles, M., and Vaadia, E. (1998). Physiological aspects of information processing in the basal ganglia of normal and Parkinsonian primates. Trends Neurosci. 21, 32-38.

Buford, J. A., Inase, M., and Anderson, M.E. (1996). Contrasting locations of pallidal-receiving neurons and microexcitable zones in primate thalamus. J. Neurophysiol. 75, 1105-1116.

stimulation (DBS), for treatment of movement disorders aimed at the motor territory of the basal ganglia, such as the GPi and STN. The somatotopy gives us useful indices to identify the targets during stereotaxic surgery (Kaplitt et al., 2003). STN-DBS sometimes induces side effects of mood changes. This may be explained by the current spread from the motor territory to the limbic and prefrontal territories of the STN because of the small size of the STN. On the other hand, GP-DBS does not induce psychological side effects, probably because the motor territory is remotely located from the limbic and prefrontal territories in the GPi.

\section{CONCLUSION}

In this article, I have described that each nucleus of the basal ganglia shows clear somatotopic organization, and that information related to different body parts is processed independently through the cortico-basal ganglia loop. I would like to point out the following unsolved questions: In the topographic projections from one nucleus to another nucleus, what kind of information is added? What kind of information is originated? How do converging inputs from multiple motor cortices contribute to the execution of voluntary movements? How is the somatotopy in each nucleus of the basal ganglia organized during development? These are important questions closely related to the functions of the basal ganglia. The somatotopic perspective way of view will be a good clue and guide for further understanding of the basal ganglia.

\section{ACKNOWLEDGMENTS}

Studies in the author's laboratory were supported by Grants-in-Aid for Scientific Research from the Ministry of Education, Culture, Sports, Science, and Technology of Japan. The author is grateful to the current and former collaborators.

Carpenter, M. B., Whittier, J. R., and Mettler, F.A. (1950). Analysis of choreoid hyperkinesia in the Rhesus monkey; surgical and pharmacological analysis of hyperkinesia resulting from lesions in the subthalamic nucleus of Luys. J. Comp. Neurol. 92, 293-331.

Chiken, S., Shashidharan, P., and Nambu, A. (2008). Cortically evoked longlasting inhibition of pallidal neurons in a transgenic mouse model of dystonia. J. Neurosci. 28, 13967-13977.

DeLong, M. R. (1971). Activity of pallidal neurons during movement. $J$. Neurophysiol. 34, 414-427.

DeLong, M. R., Crutcher, M. D., and Georgopoulos, A. P. (1983). Relations between movement and single cell discharge in the substantia nigra of the behaving monkey. J. Neurosci. 3, 1599-1606.

DeLong, M. R., Crutcher, M. D., and Georgopoulos, A. P. (1985). Primate globus pallidus and subthalamic nucleus: functional organization. $J$. Neurophysiol. 53, 530-543.

Filion, M., Tremblay, L., and Bedard, P. J. (1988). Abnormal influences of passive limb movement on the activity of globus pallidus neurons in Parkinsonian monkeys. Brain Res. 444, 165-176.

Flaherty,A.W., and Graybiel,A. M. (1993). Two input systems for body representations in the primate striatal matrix: experimental evidence in the squirrel monkey. J. Neurosci. 13, 1120-1137.

Gage, G. J., Stoetzner, C. R., Wiltschko, A. B., and Berke, J. D. (2010). Selective activation of striatal fast-spiking interneurons during choice execution. Neuron 67, 466-479.

Georgopoulos, A. P., DeLong, M. R., and Crutcher, M. D. (1983). Relations between parameters of step-tracking movements and single cell discharge in the globus pallidus and subthalamic nucleus of the behaving monkey. $J$. Neurosci. 3, 1586-1598.

Graybiel,A.M. (1990). Neurotransmitters and neuromodulators in the basal ganglia. Trends Neurosci. 13, 244-254.

Haber, S. N., Kim, K. S., Mailly, P., and Calzavara, R. (2006). Reward-related cortical inputs define a large striatal region in primates that interface with 
associative cortical connections, providing a substrate for incentive-based learning. J. Neurosci. 26, 8368-8376.

Haber, S. N., Lynd, E., Klein, C., and Groenewegen, H. J. (1990). Topographic organization of the ventral striatal efferent projections in the rhesus monkey: an anterograde tracing study. J. Comp. Neurol. 293, 282-298.

Hamada, I., and DeLong, M. R. (1992). Excitotoxic acid lesions of the primate subthalamic nucleus result in transient dyskinesias of the contralateral limbs. J. Neurophysiol. 68, 1850-1858.

Hamada, I., Delong, M. R., and Mano, N. (1990). Activity of identified wrist-related pallidal neurons during step and ramp wrist movements in the monkey. J. Neurophysiol. 64, 1892-1906.

Hikosaka, O., Sakamoto, M., and Usui, S. (1989). Functional properties of monkey caudate neurons. I. Activities related to saccadic eye movements. $J$. Neurophysiol. 61, 780-798.

Hikosaka, O., and Wurtz, R. H. (1983). Visual and oculomotor functions of monkey substantia nigra pars reticulata. I. Relation of visual and auditory responses to saccades. J. Neurophysiol. 49, 1230-1253.

Holsapple, J. W., Preston, J. B., and Strick, P. L. (1991). The origin of thalamic inputs to the "hand" representation in the primary motor cortex. J. Neurosci. 11, 2644-2654.

Hoover, J. E., and Strick, P. L. (1993). Multiple output channels in the basal ganglia. Science 259, 819-821.

Hoover, J. E., and Strick, P. L. (1999). The organization of cerebellar and basal ganglia outputs to primary motor cortex as revealed by retrograde transneuronal transport of herpes simplex virus type 1. J. Neurosci. 19, 1446-1463.

Inase, M., Tokuno, H., Nambu, A., Akazawa, T., and Takada, M. (1999). Corticostriatal and corticosubthalamic input zones from the presupplementary motor area in the macaque monkey: comparison with the input zones from the supplementary motor area. Brain Res. 833, 191-201.

Jones, E. G. (2007). The Thalamus. New York: Cambridge University Press.

Kaneda, K., Nambu, A., Tokuno, H., and Takada, M. (2002). Differential processing patterns of motor information via striatopallidal and striatonigral projections. J. Neurophysiol. $88,1420-1432$

Kaplitt, M. G., Hutchinson, W. D., and Lozano, A. M. (2003). "Target localization in movement disorders surgery," in Surgical Treatment of Parkinson's Disease and Other Movement
Disorders, eds D. Tarsy, J. L. Vitek, and A. M. Lozano (Totowa: Humana Press), 87-98.

Kita, H., Nambu, A., Kaneda, K., Tachibana, Y., and Takada, M. (2004). Role of ionotropic glutamatergic and GABAergic inputs on the firing activity of neurons in the external pallidum in awake monkeys. J. Neurophysiol. 92 , 3069-3084.

Kitano, H., Tanibuchi, I., and Jinnai, K. (1998). The distribution of neurons in the substantia nigra pars reticulata with input from the motor, premotor and prefrontal areas of the cerebral cortex in monkeys. Brain Res. 784, 228-238.

Künzle, H. (1975). Bilateral projections from precentral motor cortex to the putamen and other parts of the basal ganglia. An autoradiographic study in Macaca fascicularis. Brain Res. 88, 195-209.

Matsuda, W., Furuta, T., Nakamura, K.C., Hioki, H., Fujiyama, F., Arai, R., and Kaneko, T. (2009). Single nigrostriatal dopaminergic neurons form widely spread and highly dense axonal arborizations in the neostriatum. J. Neurosci. $29,444-453$.

Matsumura, M., Kojima, J., Gardiner, T. W., and Hikosaka, O. (1992). Visual and oculomotor functions of monkey subthalamic nucleus. J. Neurophysiol. 67, 1615-1632.

McFarland, N. R., and Haber, S. N. (2000). Convergent inputs from thalamic motor nuclei and frontal cortical areas to the dorsal striatum in the primate. J. Neurosci. 20, 3798-3813.

Miyachi, S., Lu, X., Imanishi, M., Sawada, K., Nambu, A., and Takada, M. (2006). Somatotopically arranged inputs from putamen and subthalamic nucleus to primary motor cortex. Neurosci. Res. 56, 300-308.

Monakow, K.H., Akert, K., and Künzle, H. (1978). Projections of the precentral motor cortex and other cortical areas of the frontal lobe to the subthalamic nucleus in the monkey. Exp. Brain Res. $33,395-403$.

Nakano, K., Hasegawa, Y., Tokushige, A., Nakagawa, S., Kayahara, T., and Mizuno, N. (1990). Topographical projections from the thalamus, subthalamic nucleus and pedunculopontine tegmental nucleus to the striatum in the Japanese monkey, Macaca fuscata. Brain Res. 537, 54-68.

Nambu, A., Kaneda, K., Tokuno, H., and Takada, M. (2002a). Organization of corticostriatal motor inputs in monkey putamen. J. Neurophysiol. 88, 1830-1842.

Nambu, A., Tokuno, H., and Takada, M. (2002b). Functional significance of the cortico-subthalamo-pallidal 'hyperdirect' pathway. Neurosci. Res. 43, 111-117.

Nambu, A., Takada, M., Inase, M., and Tokuno, H. (1996). Dual somatotopical representations in the primate subthalamic nucleus: evidence for ordered but reversed body-map transformations from the primary motor cortex and the supplementary motor area. J. Neurosci. 16, 2671-2683.

Nambu, A., Tokuno, H., Hamada, I., Kita, H., Imanishi, M., Akazawa, T., Ikeuchi, Y., and Hasegawa, N. (2000). Excitatory cortical inputs to pallidal neurons via the subthalamic nucleus in the monkey. J. Neurophysiol. 84 289-300.

Nambu, A., Tokuno, H., Inase, M., and Takada,M.(1997).Corticosubthalamic input zones from forelimb representations of the dorsal and ventral divisions of the premotor cortex in the macaque monkey: comparison with the input zones from the primary motor cortex and the supplementary motor area. Neurosci. Lett. 239, 13-16.

Nambu, A., Yoshida, S., and Jinnai, K. (1990). Discharge patterns of pallidal neurons with input from various cortical areas during movement in the monkey. Brain Res. 519, 183-191.

Nambu, A., Yoshida, S., and Jinnai, K. (1991). Movement-related activity of thalamic neurons with input from the globus pallidus and projection to the motor cortex in the monkey. Exp. Brain Res. 84, 279-284.

Parent, A. (1990). Extrinsic connections of the basal ganglia. Trends Neurosci. 13, 254-258.

Parent, A., and Hazrati, L. N. (1995). Functional anatomy of the basal ganglia. I. The cortico-basal gangliathalamo-cortical loop. Brain Res. Rev. 20, 91-127.

Parent,A., Mackey, A., and De Bellefeuille, L. (1983). The subcortical afferents to caudate nucleus and putamen in primate: a fluorescence retrograde double labeling study. Neuroscience 10, 1137-1150.

Percheron, G., and Filion, M. (1991) Parallel processing in the basal ganglia: up to a point. Trends Neurosci. $14,55-59$.

Percheron, G., Francois, C., Yelnik, J., Fenelon, G., and Talbi, B. (1994). "The basal ganglia related system of primates: Definition, description and information analysis," in The Basal Ganglia IV: New Ideas and Data on Structure and Function, eds G. Percheron, J. S. McKenzie, and J. Feger (New York: Plenum Press), 3-20.

Percheron, G., Yelnik, J., and Francois, C. (1984).A Golgi analysis of the primate globus pallidus. III. Spatial organiza- tion of the striato-pallidal complex. $J$. Comp. Neurol. 227, 214-227.

Pessiglione, M., Guehl, D., Rolland, A. S., Francois, C., Hirsch, E. C., Feger, J., and Tremblay, L. (2005). Thalamic neuronal activity in dopamine-depleted primates: evidence for a loss of functional segregation within basal ganglia circuits. J. Neurosci. 25, 1523-1531.

Picard, N., and Strick, P. L. (2001). Imaging the premotor areas. Curr. Opin. Neurobiol. 11, 663-672.

Sadikot, A. F., Parent, A., Smith, Y., and Bolam, J. P. (1992). Efferent connections of the centromedian and parafascicular thalamic nuclei in the squirrel monkey: a light and electron microscopic study of the thalamostriatal projection in relation to striatal heterogeneity. J. Comp. Neurol. 320, 228-242.

Schultz, W., and Romo, R. (1990). Dopamine neurons of the monkey midbrain: contingencies of responses to stimuli eliciting immediate behavioral reactions. J. Neurophysiol. 63, 607-624.

Selemon, L. D., and Goldman-Rakic, P. S. (1985). Longitudinal topography and interdigitation of corticostriatal projections in the rhesus monkey. J. Neurosci. 5, 776-794.

Smith, Y., and Parent, A. (1986). Differential connections of caudate nucleus and putamen in the squirrel monkey (Saimiri sciureus). Neuroscience 18, 347-371.

Strick, P. L., Dum, R. P., and Picard, N. (1995). "Macro-organization of the circuits connecting the basal ganglia with the cortical motor areas," in Models of Information Processing in the Basal Ganglia, eds J. C. Houck, J. L. Davis, and D. G. Beiser (Cambridge: MIT Press), 117-130.

Tachibana, Y., Kita, H., Chiken, S., Takada, M., and Nambu, A. (2008). Motor cortical control of internal pallidal activity through glutamatergic and GABAergic inputs in awake monkeys. Eur. J. Neurosci. 27, 238-253.

Tachibana, Y., Nambu, A., Hatanaka, N., Miyachi, S., and Takada, M. (2004). Input-output organization of the rostral part of the dorsal premotor cortex, with special reference to its corticostriatal projection. Neurosci. Res. 48, 45-57.

Takada, M., Nambu, A., Hatanaka, N., Tachibana, Y., Miyachi, S., Taira, M., and Inase, M. (2004). Organization of prefrontal outflow toward frontal motor-related areas in macaque monkeys. Eur. J. Neurosci. 19, 3328-3342. Takada, M., Tokuno, H., Hamada, I., Inase, M., Ito, Y., Imanishi, M., Hasegawa, N., Akazawa, T., Hatanaka, N., and Nambu, A. (2001). Organization of 
inputs from cingulate motor areas to basal ganglia in macaque monkey. Eur. J. Neurosci. 14, 1633-1650.

Takada, M., Tokuno, H., Nambu, A., and Inase, M. (1998a). Corticostriatal input zones from the supplementary motor area overlap those from the contra- rather than ipsilateral primary motor cortex. Brain Res. 791, 335-340.

Takada, M., Tokuno, H., Nambu, A., and Inase, M. (1998b). Corticostriatal projections from the somatic motor areas of the frontal cortex in the macaque monkey: segregation versus overlap of input zones from the primary motor cortex, the supplementary motor area, and the premotor cortex. Exp. Brain Res. 120, 114-128.

Tepper, J. M., Wilson, C. J., and Koos, T. (2008). Feedforward and feedback inhibition in neostriatal GABAergic spiny neurons. Brain Res. Rev. 58, 272-281.
Tokuno, H., Inase, M., Nambu, A., Akazawa, T., Miyachi, S., and Takada, M. (1999). Corticostriatal projections from distal and proximal forelimb representations of the monkey primary motor cortex. Neurosci. Lett. 269, 33-36.

Vitek, J. L., Ashe, J., DeLong, M. R., and Alexander, G. E. (1994). Physiologic properties and somatotopic organization of the primate motor thalamus.J. Neurophysiol. 71, 1498-1513.

Vitek, J. L., Ashe, J., DeLong, M. R., and Kaneoke, Y. (1996). Microstimulation of primate motor thalamus: somatotopic organization and differential distribution of evoked motor responses among subnuclei. J. Neurophysiol. 75, 2486-2495.

Vitek, J. L., Chockkan, V., Zhang, J. Y., Kaneoke, Y., Evatt, M., DeLong, M. R., Triche, S., Mewes, K., Hashimoto, T., and Bakay, R. A. (1999). Neuronal activity in the basal ganglia in patients with generalized dystonia and hemiballismus. Ann. Neurol. 46, 22-35.

Wichmann, T., Bergman, H., and DeLong, M.R.(1994). The primate subthalamic nucleus. I. Functional properties in intact animals. J. Neurophysiol. 72, 494-506.

Wichmann, T., and Kliem, M. A. (2004). Neuronal activity in the primate substantia nigra pars reticulata during the performance of simple and memory-guided elbow movements. J. Neurophysiol. 91, 815-827.

Yelnik, J., Percheron, G., and Francois, C. (1984). A Golgi analysis of the primate globus pallidus. II. Quantitative morphology and spatial orientation of dendritic arborizations. J. Comp. Neurol. 227, 200-213.

Yoshida, S., Nambu, A., and Jinnai, K. (1993). The distribution of the globus pallidus neurons with input from various cortical areas in the monkeys. Brain Res. 611, 170-174.

Conflict of Interest Statement: The author declares that the research was conducted in the absence of any commercial or financial relationships that could be construed as a potential conflict of interest.

Received: 31 August 2010; accepted: 31 March 2011; published online: 20 April 2011.

Citation: Nambu A (2011) Somatotopic organization of the primate basal ganglia. Front. Neuroanat. 5:26. doi: 10.3389/ fnana.2011.00026

Copyright (c) 2011 Nambu. This is an openaccess article subject to a non-exclusive license between the authors and Frontiers Media $S A$, which permits use, distribution and reproduction in other forums, provided the original authors and source are credited and other Frontiers conditions are complied with. 